\title{
Reflexiones sobre la investigación de procesos de salud enfermedad de los trabajadores de la salud'
}

\author{
Graciela Zaldúa \\ Psicóloga - Universidad Nacional de La Plata \\ Especialista en Planificación en Salud-CENDES - UCV (Venezuela) \\ Profesora Titular Regular de las materias Psicología Preventiva y Epidemiologia \\ - Carrera de Psicología - Universidad de Buenos Aires (UBA) \\ Profesora de la Maestria en Psicologia Social Comunitaria - UBA \\ Directora del proyecto de investigación UBACyT "Praxis Psicosocial \\ Comunitaria en Salud" Investigadora UBACyT Categoría 1. \\ E-mail: gzaldua@psi.uba.ar
}

M. Marcela BottinelloI

Lic. en Psicología - Universidad de Buenos Aires

(UBA)

Magister en Metodología de la Investigación

Científica - Universidad Nacional de Lanús

(tesis en trámite)

Doctoranda en Salud Mental Comunitaria -

Universidad Nacional de Lanús. Posgrado

Internaçional organizado por Red Martistán

y subsidiado por Programa Alfa

Profesora titular de la cátedra de Metodologia

de la Investigación y Estadistica en la Carrera

de Terapia Ocupacional, UBA.

Co-directora del proyecto de investigación

UBACyT "Praxis Psicosocial Comunitaria

en Salud"

Docente de grado y posgrado. Asesora, directora y evaluadora de becarios, tesis $y$ proyectos.

E-mail: mbottine@psi.uba.ar

Mariela Nabergol

Licenciada en Terapia Ocupacional,

Universidad de Buenos Aires (UBA).

Docente de la cátedra de Metodologia de la

Investigación y Estadística en la Carrera de

Terapia Ocupacional, UBA.

Integrante del equipo de investigación UBACyT

"Praxis Psicosocial Comunitaria en Salud"

Becaria de doctorado UBACyT.

Doctoranda en Salud Menfal Comunitaria.

Universidad Nacional de Lanús (UNLa).

Asesora y evaluadora de tesis de licenciatura

en terapia ocupacional.

E-mail: mnabergoi@yahoo.com.ar

\section{Resumen}

El caracteriza el síndrome de burnout como eje para pensar las articulaciones entre reflexividad, investigación y práctica en el ámbito de las relaciones salud-trabajo. Tomando como experiencia el trabajo desarrollado por nuestro equipo de investigación de la Universidad de Buenos Aires, se reflexiona sobre las características del estrés asistencial en los trabajadores de la salud y su especificidad en terapia ocupacional. Se sostiene la necesidad de realizar una reflexión epistemológico-metodológica para pensar las relaciones existentes entre los diferentes contextos de producción de las investigaciones y de los propios trabajadores de salud, incluyendo el marco histórico y político-económico como

\footnotetext{
${ }^{1}$ Nota: este artículo ha sido parcialmente publicado en el libro "Saude, Trabaltho e Terapia Ocupacionar" Lancman, Selma (Org.). Ed. Roca. San Paulo, Brasil. 2004
} 
determinante en la configuración del sindrome y sobre las herramientas utilizadas para medirlo e interpretarlo.

Desde una postura crítica y comprometida en la necesidad de trabajar integralmente la teoría y la práctica se propone realizar una mirada reflexiva sobre las conceptualizaciones y sus implicancias en el campo de los procesos de salud-enfermedad que posibilite salir de las posiciones dicotómicas $y / u$ objetivantes de sus prácticas en salud como prácticas que se realizan sobre el polo negativo y no sobre las potencialidades.

\section{Palabras claves}

Sindrome de Burnout- reflexividad- Trabajadores de la salud- investigacióninterdisciplina-salud colectiva- Terapia Ocupacional

The Burnout Syndrome is characterized as an axis to think on the relations between reflexivity, research and practice in the field of work-health relations. Based on the work developed by our research team at Buenos Aires University (UBA) we reflect on the characteristics of assistential stress in health workers and is specificity in occupational therapy. It sustains the necessity of working in an epistemological-methodological reflection to think the existing relations between the different production contexts of research and the health workers themselves, including the historical and ecocomical-political framework as determining the syndrome's configuration and about the research tools used to measure and interpret it.

From a critical posture, compromised with the necesity of working integrally theory and practice, it is proposed a reflective sight on the conceptualizations and their implications in the field of the health-illness processes that make able to get out of the dichotomic and/or objetivizing positions of their health practices as practices that are done on the negative pole and not on the potentialities.

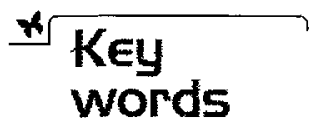

Burnout Sindrome - reflexivity - health workers - researcj - interdiscipline - collective health- occupational therapy 
El trabajo tiene como objetivo presentar algunos aspectos del síndrome de burnout como problemática vinculada al trabajo en salud en general y más específicamente al trabajo de los terapeutas ocupacionales.

El mismo se realiza desde la experiencia obtenida en el marco de la investigación del proyecto UBACyT 2001-2003 "Estudio sobre las Condiciones de Producción de los Actos de Salud en Hospitales Públicos" (P065), la Cátedra de Metodología de Investigación y Estadistica de la Carrera de Terapia Ocupacional (Facultad de Psicología, Universidad de Buenos Aires) y la beca de investigación UBACyT 2001 "Caracterización del Perfil Ocupacional de los Trabajadores de la Salud de Hospitales Públicos".

Este trabajo busca interpelar a los terapeutas ocupacionales en dos modos diferentes y complementarios a la vez: 1) como profesional cuyo desempeño ocupacional implica la implementación de estrategias salutíferas y 2) como trabajador cuya salud se ve afectada por su propia inserción en el proceso productivo. Pretende así promover la reflexión de los terapeutas ocupacionales sobre las condiciones de producción de sus prácticas y el modo en que éstas afectan su salud y propiciar a su vez la identificación e implementación de estrategias salutiferas individuales y colectivas en los diferentes ámbitos de trabajo.

\section{¿Qué es el síndrome de burnout?}

Se llama sindrome de burnout ${ }^{\dagger}$ a un estrés laboral crónico propio de los profesionales de servicios humanos, cuya tarea implica una atención intensa y prolongada con personas que están en situación de necesidad o de dependencia. El mismo tiene como componentes principales la fatiga emo- cional, la despersonalización y una sensación de reducido logro personal que aparecen como respuesta a una tensión emocional de índole crónica, originada por el deseo de lidiar exitosamente con otros seres humanos que tienen problemas. 
Burnout es una palabra de origen inglés que quiere decir "fundido" o "quemado". Así lo denominó por primera vez el investigador $\mathrm{H}$. Freudenberger en la década del '70 en EEUU a partir de la observación de que "muchos de los trabajadores de la institución (de salud) en la que él se desempeñaba presentaban un gran desgaste emocional, pérdida de motivación y compromiso hacia el trabajo"z. El concepto fue luego sistematizado por las investigadoras Cristina Maslach (Profesora de la Universidad de California, investigadora en psicología social y de la salud) y Susan Jackson, quienes se interesaron por el tema al encontrar correspondencias con los resultados de su investigación con trabajadores de cuidados intensivos y desarrollaron un instrumento para medir el fenómeno, el Maslach Burnout Inventory (MBI), que ha sido traducido a diversos idiomas y utilizado mundialmente en las últimas décadas.

Desde estas primeras aproximaciones, el burnout ha sido estudiado principalmente como característico de diversos colectivos de trabajadores de la salud, aunque en los últimos años se ha ampliado también a otras profesiones de servicios humanos.

Su estudio por parte de diversos investigadores ha derivado también en diferentes aproximaciones y explicaciones del fenómeno.

Algunos enfoques to han descripto como una entidad clinica, de carácter individual, clasificando su curso, sintomatología, etiologia y diagnóstico diferencial. En ellos cobran especial importancia las variables sociodemo- gráficas tales como la edad, el genero, el tipo de personalidad y las características de la formación profesional. Otros (Gil Monte, Peiró, Martínez-Guerra), en cambio, han privilegiado un enfoque psicosocial donde adquieren mayor importancia los elementos laborales y organizacionales.

Pero si bien no existe actualmente una definición del burnout universalmente aceptada, encontramos algunos puntos básicos de acuerdo. Por una parte que en tanto estrés, el burnout comparte características con otros tipos de estrés. Su particularidad, sin embargo, está dada por su carácter crónico y por describir una respuesta al estrés laboral característica de las profesiones "de ayuda". Por otra, que el burnout no es un punto estático ni un estado terminal sino más bien un proceso creciente que se instala en forma paulatina.

Maslach y Jackson, por ejemplo, describen el burnout como un proceso que atraviesa tres fases ${ }^{1}$. La primera fase es de Cansancio Emocional, que se refiere al sentimiento de estar sobrecargado, sobrepasado y agotado emocionalmente por el trabajo. Esta sobreviene luego de haber intentado modificar las situaciones estresantes y habiendo fracasado en la gestión. La segunda corresponde a la Despersonalización. La despersonalización es una defensa que la persona construye para protegerse frente a los sentimientos de impotencia, indefensión y desesperanza. Indica una respuesta impersonal y carente de sentimiento hacia los pacientes y colegas y puede incluir el desarrollo de actitudes hostiles hacia ellos. Finalmente acaece el 
Abandono de la Realización Personal. El profesional sucumbe a la creencia de que el trabajo no amerita más esfuerzos. Se presenta como un sentimiento de incompetencia y falta de efectividad en el trabajo e incluye diversas evaluaciones negativas de uno mismo.

Otra investigación ha identificado el proceso de burnout como atravesando cuarto niveles ${ }^{3}$. Un primer nivel, leve, está caracterizado por la presencia de quejas recurrentes, cefaleas, dolores de espalda, síntomas vagos, cambios en el carácter, cansancio y dificultad para levantarse a la mañana. En el nivel moderado aparecen ya insomnio, dificultad para concentrarse, dificultad en las relaciones interpersonales, disminución de la libido, aislamiento, negativismo. A medida que el proceso sigue su curso, en el nivel denominado grave encontramos aumento del absentismo, disminución notable en la productividad, sensación de disgusto, rechazo o aversión, baja autoestima, automedicación con psicofármacos y abuso de alcohol u otras drogas. Finalmente, en el nivel extremo pueden aparecer un marcado aislamiento, crisis existencial, sensación de fracaso, colapso, riesgo suicida importante y/o cuadros psiquiátricos. Encontramos esta clasificación particularmente útil para la identificación de "señales de alarma" para realizar intervenciones en el sentido de evitar la progresión hacia los niveles más comprometidos.

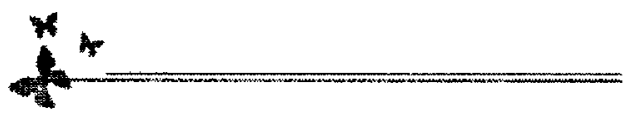

Las estrategias de afrontamiento ¿Cómo prevenir y abordar el sindrome de burnout?

Dentro de las medidas de prevención generales descritas en la literatura se incluyen las estrategias de afrontamiento del estrés ${ }^{4}$ : reducir las tensiones del ambiente de trabajo, recibir una mejor remuneración, regular los tiempos de descanso, realizar deportes, controlar el peso y las salud en general.

Específicamente en relación con el burnout se han propuesto diversas estrategias tanto desde la reflexión teórica como del trabajo con los propios trabajadores de salud que han operado promoviendo el polo saludable de los trabajadores. En algunas de ellas, la terapia ocupacional como disciplina específica de la ocupación humana puede jugar un papel protagónico.

A nivel individual algunos autores recomiendan tener una terapia psicológica individual ${ }^{5}$. Pero también se enfatiza la importancia de poder establecer una rutina de "descompresión laboral" mediante la realización de actividades de esparcimiento y recreativas $^{4}$. A nivel de la organización es muy importante mantener contacto con otros profesionales, compartir experiencias, fomentar espacios de reflexión y transformación tanto formales como informales, y actualizarse ${ }^{5}$. También se menciona el trabajar para tener un buen funcionamiento en el equipo, poder establecer objetivos en común y organizar de manera positiva el espacio físico y el ambiente de trabajo, entre otros. 
Poniendo énfasis en el polo de la salud, Zaldúa ${ }^{2}$ trabaja con los conceptos de resiliencia y sistemas de apoyo. El primero es desarrollado por Stefan Vanistendae $\left.\right|^{6} \mathrm{e}$ incluye los dos componentes presentados en el párrafo anterior: uno consiste en la resistencia frente a la destrucción (la capacidad de proteger la propia vida aún estando bajo presión); el otro se refiere a la capacidad de construir una respuesta vital positiva frente a circunstancias dificiles. Los sistemas de apoyo, concepto elaborado por Caplan ${ }^{2}$ son aquellos que refuerzan o construyen to que él denomina recursos de competencia. Estos recursos de competencia son los que le permiten a un sujeto afrontar situaciones de riesgo.

Entre los diferentes tipos de apoyo social, House ${ }^{2}$ ha sistematizado: 1) el apoyo emocional, relacionado con el afecto y la confianza, 2) el apoyo instrumental que incluye los recursos materiales, económicos o de servicios con los que contamos, 3) el apoyo informativo, que le permite a una persona conocer una situación y poseer instrumentos para afrontarla y 4) el apoyo evaluativo, vinculado con el feedback que se tiene de las propias acciones, y también con la posibilidad de obtener reconocimiento por la tarea que se realiza.

Como no se pudo comprobar una relación mecánica entre el trabajo y la descompensación psíquica, la psico- patología del trabajo comenzó a centrar su atención en la normalidad?. Para Dessors y Moliner -integrantes del equipo de Dejours-, si bien trabajar significa enfrentarse cotidianamente a cuestiones peligrosas para la salud mental del trabajador, lo cierto es que en general la mayoría de los trabajadores no se vuelven "locos". Sin embargo, el trabajo produce sufrimiento psiquico en el trabajador y lo lleva a construir defensas individuales y colectivas que tienen un alto costo energético para su mecanismo psíquico.

Pensar en implementar estrategias de afrontamiento implicaria entonces crear condiciones de posibilidad para que estas cosas con las cuales se enfrenta el terapeuta ocupacional como trabajador tengan el menor costo posible para su salud y la de sus compañeros de trabajo. $Y$ para sus pacientes, porque el fenómeno que describimos influye en gran medida en la calidad de atención que puede brindar. Pero también implicaría trabajar descle los aspectos resilientes (aquellos que permiten a las personas no sólo enfrentarse y sobreponerse sino también ser fortalecido o transformado por experiencias de adversidad ${ }^{8}$ hacia lo salutífero. Desde terapia ocupacional esto supondrá poner en juego la capacidad de transformar el modo en que los trabajadores desempeñamos nuestras ocupaciones tanto en sus dimensiones individuales como colectivas.
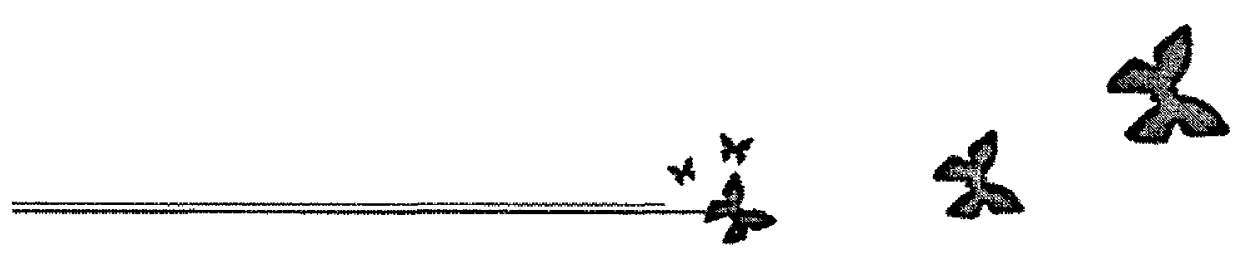


\section{El síndrome de burnout en el proceso de la terapia ocupacional}

Se han realizado algunas investigaciones sobre burnout en terapeutas ocupacionales en Estados Unidos y Australia en la década del ' 80 , luego de que en el año 1982 la AOTA incluyera un taller de prevención de burnout en su conferencia anual. Estas investigaciones (Strgess \& Poulsen, 1983'; Broiller, Bender, Cyranowski y Velletri, 1986-1987 10-11; Rogers y Donson, $1988^{12}$ ) asi como la realizada en Brasil en 1995 por la TO Lilian Magalhaes ${ }^{13}$ buscaban "comprender el por qué del notorio abandono de la profesión por parte de muchos colegas y/o su dedicación predominante a tareas docentes y/o administrativas, dejando a un lado la dedicación clínica en los primeros años de su carrera"14.

En estos estudios se citan factores propios de la profesión que pueden tanto aumentar como disminuir la posibilidad de sufrir burnout.

Entre los primeros se destacan:

- la severidad del diagnóstico y pronóstico de los pacientes

- la escasa valoración y reconocimiento social de la profesión

- la discriminación de género

- la ambigüedad del rol y la inseguridad del terapeuta ocupacional en el trabajo en equipo

- la discrepancia entre las expectativas generadas por la formación de grado y la realidad en relación con los resultados de la intervención con pacientes crónicos

- el énfasis que el sistema norteamericano pone en la productividad y rentabilidad de sus servicios.

Teniendo en cuenta las subcategorias del sindrome descriptas más arriba (Cansancio Emocional, Despersonalización y Abandono de la Realización Personal), es interesante notar que, a excepción del último, los factores enumerados pueden favorecer niveles más elevados de burnout en la categoria Abandono de la Realización Personal en tanto contribuyen a la construcción de un sentimiento de inefectividad, tal como se refleja en los resultados del estudio de Rogers y Donson" en el que los niveles de burnout en esta categoria fueron más elevados que en las restantes. Estas autoras sugieren que éste es el componente del burnout al que los terapeutas ocupacionales serían más susceptibles.

Pero el proceso de terapia ocupacional tendría también características propias que promueven procesos salutíferos frente a este fenómeno. Así, la autonomía y creatividad -dos características cuya ausencia se asocia a mayores niveles de Cansancio Emocional-requeridos para la para la elección y adaptación de actividades apropiadas para el tratamiento junto con la singularidad de los casos atendidos 
permitirian revitalizar las reservas emocionales y construir defensas contra el cansancio emocional. Por otro lado, el hecho de que el proceso de terapia ocupacional requiera la cooperación activa del paciente como colaborador en el tratamiento y la posibilidad de sostener el tratamiento por un período de tiempo prolongado ofrecerían oportunidades para una mayor interacción personal, promoviendo un menor nivel de despersonalización.

Aun cuando las investigaciones citadas impiden sacar conclusiones fuertes debido a que son demasiado escasas y tienen resultados a veces contradictorios ${ }^{15}$, se pueden observar similitudes y diferencias entre la realidad que describen los estudios efectuados en EEUU y Australia, y la situación de los terapeutas ocupacionales de Brasil y Argentina. Acerca de los resultados de la investigación realizada en Brasil, la autora dice:

"Paradójicamente, ningún formulario mencionó aspectos relacionados con la relación específica con los clientes. Lo que incomoda a los terapeutas es la escasez de recursos, la competencia con el equipo, la falta de respeto y consideración por parte de los demás profesionales de la salud, la burocracia excesiva y hasta la inoperancia de la entidades de clases. Ninguna palabra fue dicha sobre las dificultades eventualmente impuestas por el cliente, dada la complejidad de la clínica u otros aspectos semejantes"12.

Estos resultados coinciden con los obtenidos como parte de la investigación
"Estudio sobre el burnout en Hospitales Públicos"16 realizada en Buenos Aires, Argentina, en una sub-muestra conformada por 10 terapeutas ocupacionales de un hospital psiquiátrico. En la misma, las respuestas a una pregunta abierta sobre las mayores causas de malestar en el trabajo tampoco se hace mención a relación específica con los pacientes/clientes. Como en las otras investigaciones citadas, se mencionan aspectos relacionados con la falta de reconocimiento social y las dificultades del trabajo en equipo (ej.: el "poco respeto y reconocimiento de los otros", "la competitividad", el "clima laboral", "el poco interés en el trabajo interdisciplinario"). Sin embargo, las respuestas priorizan como causas de malestar en el trabajo aspectos ideológicos, políticos e institucionales como son la "desorganización institucional", "las condiciones burocráticas", la "falta de voluntad politica", la "desarticulación de las acciones", las "diferentes ideologías en relación con el proceso salud-enfermedad", entre otras respuestas similares.

A diferencia de las investigaciones realizadas sobre burnout en terapeutas ocupacionales, las cuales buscaban explicar la deserción profesional de nuestros colegas, las investigaciones actuales sobre burnout en el campo de la salud en Argentina parecen intentar responder a la necesidad de los profesionales del área de comprender, explicar y denunciar las condiciones de trabajo en las que están insertos, haciendo un llamado de atención para pensar sobre su propia salud. 
Estos hallazgos se vinculan estrechamente con nuestra experiencia de investigación en otros colectivos de trabajadores de salud. Las diferencias obtenidas en los resultados arrojados por el MBI con respecto a aquellos que arrojan las metodologias cualitativas que utilizamos nos han llevado a considerar imprescindible la inclusión del estudio de las dimensiones históricas y político-económicas que determinan actualmente las condiciones de producción de los actos de salud ${ }^{17-18-19} \mathrm{en}$ tendiendo que estas dimensiones no solo contextualizan la práctica sino que determinan lo que ocurre dentro de las paredes del consultorio, la relación profesional / paciente, los alcances y limitaciones del tratamiento, la significación de las ocupaciones. En definitiva: que estas dimensiones son también "inherentes" al trabajo asistencial. Consideramos indispensable para comprender el fenómeno, pues, una consideración acerca del lugar en que han quedado los profesionales de la salud en el contexto de las transformaciones producidas en los últimos años en Latinoamérica por la implementación de políticas neoliberales que siguen los lineamientos de las propuestas del Banco Mundial20-21. El viraje producido hacia pensar la salud como mercado sujeto a las leyes de oferta y demanda, la tendencia a la privatización de los servicios, el predominio de la inversión en recursos tecnológicos por sobre los recursos humanos que ha producido grandes transformaciones en las formas de trabajo y los modos de trabajo en salud ${ }^{22}$.
En nuestro pais, por ejemplo, en el ámbito público, la caida del valor social de la protesión médica ( $\mathrm{y}$ de los profesionales de salud en general), la escasez de recursos en salud, la sobrecarga laboral por incremento en la demanda o por sobreempleo, la crisis salarial, la amenaza por juicios de mala praxis y los dilemas éticos que presentan las nuevas tecnologías u otros problemas emergentes frente al acto de salud $^{2}$. En el ámbito privado, las formas de contratación inestable producto de la tendencia de la subcontratación en bloque de las empresas privadas, presiones de tiempos con exigencias crecientes de productividad, disminución de los honorarios profesionales, entre otros ${ }^{17}$. Sin estas consideraciones se nos hace imposible poder comprender en profundidad el fenómeno ${ }^{23}$ y las aparentes contradicciones encontradas entre los datos que arrojan las metodologías cualitativas y aquellos que provienen de las metodologías cualitativas.

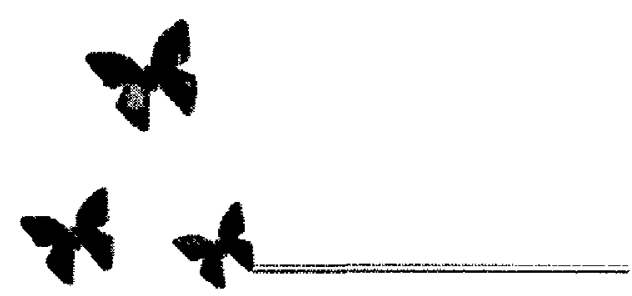




\section{Referencias y bibliografía}

1. Maslach C., Jackson S.E. 1982. Burn-out in health professions. Citado en Zaldua G., Lodieu M.T. El burnout: la salud de los trabajadores. Investigaciones en Psicología. Instituto de Investigaciones, Facultad de Psicología, UBA. Año 2001; 5 (1):151-169.

2. Zaldua G., Lodieu M.T. El burnout: la salud de los trabajadores. Investigaciones en Psicología. Instituto de Investigaciones, Facultad de Psicologia, UBA. Año 2001: 5 \{1\}:151.169.

3. Moreno-Jimenez B., Peñacoba Puente C. Estrés Asistencial en los Servicos de Salud. Citado en: Zaldua G., Lodieu M.T. El burnout: la salud de los trabajadores. Investigaciones en Psicología. Instituto de Investigaciones, Facultad de Psicologia, UBA. Año 2001; $5\{1\}: 151-169$.

4. Sbriller L. Prevención Primaria y Calidad de Vida en la Función del Terapeuta Ocupacional.

En: Introducción a Terapia Ocupacional. Marcos

conceptuales. Buenos Aires (Argentina):

Ed. Catálogos; 1997. p. 103-111

5. Martínez P. M., Guerra M. P. Síndrome de burnout: el riesgo de ser un profesional de ayuda. Revista Chilena de Medicina Social Salud $\gamma$ Cambio. Año 1997; 6(23):45-59.

6. Vanistendael S, Leonte J, Le boneur est toujours posible. Construire la résilience, Paris, Bayard Editions, 2000. Citado en: Melillo A., Suarez Ojeda E. N. (comp.) Resiliencia. Descubriendo las propias fortalezas. Buenos Aires (Argentina): Editorial Piados; 2001.p 86

7. Dessors D, Moliner P. La Psicódinámica del Trabajo. In: Ghio-Bailly MP (Comp) Organización del trabajo y salud, Asociación Trabajo y Sociedad, Programa de Investigaciones Económicas sobre Tecnología, Trabajo y Empleo (CONICET). Buenos Aires (Argentina). Ed. Lumen Humanitas; 1994.

8. HENDERSON GROTBERG, E. Introducción. Nuevas tendencies en resiliencia. In: MELILLO, A.; SUAREZ OJEDA, E. N. (comp.) Resiliencia.

Descubriendo las propias fortalezas. Buenos Aires, Editorial Piados, 2001.

9. Stugess, J.y Poulsen, A. The prevalence of burnout in occupational therapists. 0ccupational Therapy In Mental Health 1983; 3 (4):47-60.
10. Broillier C., Bender D., Cyranowski J., Velletri C. A pilot study of job burnout among hospital-based Occupational Therapists. The Occupational Journal of Research 1986; 6(5):285-299.

11. Broillier C., Bender D., Cyranowski J., Velletri C. OTR burnout: a comparison by clinical practice. Occupational Therapy In Mental Health 1987; 7 (1): 39-54

12. Rogers C.J., Dodson S.C. Burnout in occupational therapists. American Journal of Dccupational Therapy 1988 Dec; 2 (12):787-792.

13. Magalhaes L. V. La salud de los terapeutas ocupacionales. Pontificia Universidad Católica de Campinas, 1996. Traducción no autorizada de Sbriller L., Mimeo.

14. Nabergoi M. Reflexiones sobre el Síndrome de Burnout en Terapia Ocupacional. Anuario de Publicaciones $X$. Secretaría de investigaciones, Facultad de Psiçología, UBA. Año 2002: 489-493.

15. BrownG. T., Pranger, T. Predictos of burnout for psichiatric occupational therapy personel. CJOT 1992; 59 (5). Citado por Magalhaes L. V. La salud de los terapeutas ocupacionales. Op. Cit.

16. Material de la investigación UBACyT 19982000 "Estudio del Sindrome de Burnout en Hospitales Públicos", TP 057. Directora: Graciela Zaldúa. Con sede en el Instituto de Investigaciones, Facultad de Psicología, Universidad de Buenos Aires. Inédito

17. Zaldúa G., Lodieu M.T., Tisera A., Pawlowicz M. P. Las problemáticas de los trabajadores de la salud, VIl Anuario de Investigaciones, Buenos Aires, Secretaría de Investigaciones Facultad de Psicología - UBA, 2000

18. Almeida Filho N., Silva Palm J. La crisis de la salud pública y el mavimiento de salud colectiva en Latinoamérica. Cuadernos Médico Sociales 1999 May; N $^{\circ} 75$, CESS -Centro de Estudios Sanitarios y Sociales, Rosario

19. Breilh J. Nuevos conceptos y técnicas de investigación. Quito (Ecuador):

Centro de Estudios y Asesoría en Salud; 2000 
20. Laurell A.C. La salud como derecho social o mercancia, En: Fundación F, Erbert. Nuevas tendencias y altenativas en el sector salud. UAM Xochimilco (México); 1994

21. Ugalde A., Jackson, J-T. Las políticas de salud del Banco Mundial: una revisión crítica. Cuadernos Médico Sociales 1998 May;

(73) CESS

22. Stolkiner A. Tiempos posmodernos:

Procesos de ajuste y Salud Mental. En: Saidon 0., Troianovsky P. (comp.) Políticas en Salud Mental. Buenos Aires (Argentina): Lugar Editorial; 1994.

23. Bottinelli M M y cols. Metodología de la Investigación. Herramientas para un pensamiento complejo. Buenos Aires (Argentina).

El autor; 2003
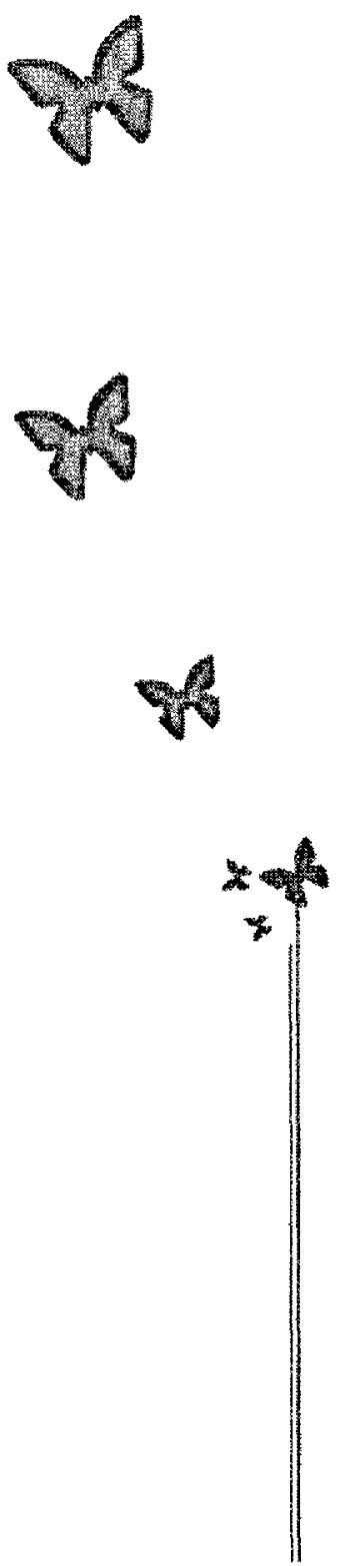